\title{
$\triangle$ RDW: A Novel Indicator with Predictive Value for the Diagnosis and Treatment of Multiple Diseases
}

Jingsheng Wang

Qiang Xiao

Yuanmin Li

Department of Cardiology, the Second Affiliated Hospital, Shandong First Medical University \& Shandong Academy of Medical Sciences, Taian, People's Republic of China
Correspondence: Yuanmin $\mathrm{Li}$ Department of Cardiology, the Second Affiliated Hospital, Shandong First Medical University \& Shandong Academy of

Medical Sciences, No. 366 Taishan Street, Taian, 271000, People's Republic of China Tel +86-135838435/8

Fax +86-538-6222036

Email Liym575@I26.com

\begin{abstract}
Elevated red blood cell distribution width (RDW) is a powerful predictor of poor prognosis in a variety of diseases, but a single measurement of RDW cannot reflect the dynamic change of diseases. $\triangle \mathrm{RDW}$, as a risk stratification tool, can be used to record changes in RDW before and after treatment; also, it allows investigators to name the unit change of RDW in the studied population. So far, there have been few relevant studies on the predictive value of $\triangle \mathrm{RDW}$ for different diseases; this article aims to review the studies and summaries of the current understandings on the correlation between $\triangle \mathrm{RDW}$ and disease outcomes.
\end{abstract}

Keywords: red cell distribution width, increment, diagnosis, prognosis

The red cell distribution width (RDW) is one of the parameters included in blood routine test, reflecting the degree of heterogeneity of red blood cell volume in peripheral blood. It has been traditionally used for differential diagnosis of anemia in laboratory tests. Evidence in the past decade attests that an elevated RDW is a common finding in human diseases such as ischemic stroke, ${ }^{1}$ Parkinsonism, ${ }^{2}$ pulmonary embolism, ${ }^{3}$ acute pancreatitis, ${ }^{4}$ acute kidney injury, ${ }^{5}$ sepsis, ${ }^{6}$ hepatitis $\mathrm{B},{ }^{7}$ chronic obstructive pulmonary disease, ${ }^{8}$ as well as in cardiovascular diseases including hypertension, carotid atherosclerosis, heart failure, atrial fibrillation, acute coronary syndrome, and stable angina. ${ }^{9-13}$ But so far, the exact mechanism of RDW abnormalities remains unclear. Previous studies have found that it was relevant to inflammatory responses, ${ }^{14}$ tumor necrosis factor $\alpha,{ }^{15}$ and oxidative stress responses. ${ }^{16}$ These conditions may influence erythropoietin production and increase RDW values. More recently, Salvagno et $\mathrm{al}^{17}$ and other scholars have provided a variety of new insights into the factors affecting RDW, such as nutritional deficiency, renal insufficiency, hepatic congestion and so on.

Currently, RDW is regarded as a predictive marker and independent risk factor for the occurrence and development of multiple diseases. ${ }^{3,18-20}$ This observation is based on a single RDW measure; however, there is no sufficient information about the effect of changes in RDW before and after treatment of disease because of a lack of attention and thorough studies. A novel concept reflecting dynamic changes of RDW, $\triangle$ RDW has been introduced recently. It can be used not only to record the difference between the baseline RDW value and the one measured at each time point after clinical treatment but also name the unit change of RDW in 
the studied population by investigators. This article reviews the studies on the correlation between $\triangle \mathrm{RDW}$ and clinical outcomes to provide relevant theoretical guidance for risk stratification and prognosis of different types of diseases.

\section{$\triangle R D W$ and Blood Transfusion}

Although red blood cell transfusion has been used as a clinical treatment for more than 100 years, ${ }^{21}$ cumulative evidence suggests that the treatment is not entirely harmless while saving lives. In fact, RBC transfusions may be potential confounders on interpretation of RDW changes. Spadaro et $\mathrm{al}^{22}$ found that $\triangle \mathrm{RDW}$ predicted the effect of blood transfusion in patients to some extent. In this prospective study, RDW's changes were documented in 36 patients (18 with respiratory failure, 9 with sepsis/septic shock, and 9 with heart failure) who were transfused with 1 unit RBC. Twenty-six (72\%) of the patients had an increased RDW level immediately after transfusion, and these patients had a higher 28-day mortality than others $(11 / 26,42 \%$ vs $1 / 10,10 \% ; p=0.06)$, with the maximum $\triangle \mathrm{RDWs}$ occurring at 24 hours post-transfusion and remaining high for at least 48 hours. The $\triangle \mathrm{RDW}$ was here defined as the difference between baseline RDW value and the value at each time point after transfusion. $\triangle \mathrm{RDW}_{\text {max }}$ was $0.55 \%$ in average per patient, and moderately correlated with the differences between $\mathrm{MCV}_{\text {donors }}$ and $\operatorname{MCV}_{\text {patient }}(r=0.478, p=0.005)$.

Based on this study, administration of exogenous RBCs can affect RDW. Patients with higher RDW values show higher mortality and transfusion requirements, while transfusion-induced $\triangle \mathrm{RDW}$ alterations also show prognostic value in critical illness. Patients with normal baseline RDW values had greater changes in $\triangle$ RDW and higher survival after transfusion. Those with higher RDW had more minor changes in $\triangle \mathrm{RDW}$ and higher mortality after transfusion, which should be related to the higher degree of erythrocyte heterogeneity in patients themselves.

\section{$\Delta R D W$ and Rheumatoid Arthritis}

In a survey study, ${ }^{15}$ it was shown that RDW levels were more significantly increased in RA patients, and RDW may be a potential marker for monitoring patients' disease progression compared with inflammation and cytokines. Both inflammatory rheumatoid arthritis (RA) and ankylosing spondylitis (AS) are chronic autoimmune inflammatory diseases, which may be used to partly explain the elevated RDW levels in RA. ${ }^{23}$ Another possible reason is that anemia occurs in approximately $60 \%$ of the patients with RA.

By comparing laboratory findings in 222 patients with RA, 150 patients with AS, and 78 patients with osteoarthritis (OA), ${ }^{24}$ it was found that $\triangle \mathrm{RDW}$ was more correlated with RA disease activity. The value of $\triangle R D W$ in RA patients was significantly greater than in healthy controls, $\mathrm{AS}$, and OA patients. The greater the variation in $\triangle \mathrm{RDW}$ values is, the higher the risk of RA is.

\section{$\triangle R D W$ and Dementia}

With the progression of modern medical technology, the average life expectancy of people is prolonged, and the incidence of dementia is also increasing year by year. In a study involving 2556 people older than 65 years, ${ }^{25}$ participants were regularly tested on RDW. They underwent cognitive assessment by a professional neurologist, including direction, attention, memory, language, and perception testing. A total of $525(20.5 \%)$ people were diagnosed with dementia by the end of the survey cycle. The researchers found that for every additional unit of RDW, the risk of developing dementia increased by about $6 \%$ (OR, 1.06; 95\% CI: 1.00-1.13). The greater the $\triangle \mathrm{RDW}$ changes, the greater the prevalence of dementia. Surprisingly, the conventional risk factors such as smoking, alcohol consumption, diabetes, and daily exercise had little effect on dementia. RDW was associated with dementia more strongly among participants without anemia (OR, 1.09; 95\% CI, 1.00-1.43) than among those with anemia (OR, 0.99; 95\% CI, 0.86-1.18), although this difference was not statistically significant. This demonstrates that $\triangle \mathrm{RDW}$ may provide new information about the risk of morbidity. Winchester et $\mathrm{al}^{26}$ proposed an inverse relationship between RDW and test results of reasoning and numerical characteristics, that is, RDW increases and cognitive ability decreases. Conversely, RDW will decrease with cognitive improvement, which is consistent with Weuve's view. ${ }^{25}$ Moreover, as RDW is increased, brain volume tends to decrease, ${ }^{27}$ which may be the structural basis for pathogenesis of dementia.

The underlying mechanism of $\triangle \mathrm{RDW}$ abnormalities in dementia remains unclear. To some extent, it may be related to the oxygen-carrying capacity of red blood cells. This difference in capacity is often the result of increased heterogeneity in red blood cell volume, which also triggers cerebrovascular hypoperfusion, leading to oxidative stress, inflammation, and neurodegeneration. ${ }^{27}$ Clearly, future research should focus on exploring the 
causal relationship between dementia and dynamic changes in RDW and underlying mechanisms, and whether intervention with RDW can improve the outcome of dementia.

\section{$\triangle R D W$ and Cerebral Infarction}

The incidence and mortality of cerebral infarction (CI) have increased significantly. The laboratory results of 392 patients with a primary diagnosis of CI suggested that RDW is an independent protective factor of carotid artery atherosclerosis (CAS) in patients with ischemic stroke. $^{28}$ Relevant studies by Hasan et $\mathrm{al}^{29}$ revealed that the incidence of CI increased when $\triangle \mathrm{RDW}$ was positively altered. The prothrombotic state was promoted by activating the renin-angiotensin system via angiotensin II type 1a receptors. This process can also boost erythropoietin levels, causing an increase in RDW. It may explain why RDW levels are higher in stroke patients. ${ }^{30,31}$

\section{$\triangle R D W$ and Community-Acquired Pneumonia}

Community-acquired pneumonia (CAP) refers to respiratory infection suffered outside the hospital, and some latent pathogen infections can develop within the average incubation period after admission. The disease occurs at a high rate between the ages of 65 and $79 .{ }^{32}$ It is estimated that in developing countries there are 151 million new episodes annually, ${ }^{33}$ and 3.5 million children die each year due to CAP morbidity worldwide. ${ }^{34}$ In the United States, approximately 20 per 1000 people older than 60 years of age have $\mathrm{CAP},{ }^{35}$ ranked as the ninth leading cause of death. According to a large-scale population survey study, ${ }^{36}$ RDW has better sensitivity than CURB-65 score in predicting intensive care unit (ICU) admission rate or mortality in patients with CAP. Lee et $\mathrm{al}^{37}$ retrospectively studied 1069 hospitalized patients with CAP. They calculated the changes in RDW during hospitalization, $\Delta \mathrm{RDW}=\left(\mathrm{RDW}_{\text {Day } 1}-\mathrm{RDW}_{\text {DayN }}\right) /$ $\mathrm{RDW}_{\text {Day } 1} \times 100 \%$, to obtain $\Delta \mathrm{RDW}_{2-1}, \Delta \mathrm{RDW}_{3-1}, \quad \ldots$ $\triangle \mathrm{RDW}_{\mathrm{N}-1}$, respectively, and followed up to determine 30-day death rate. It was found that patients with RDW $\leq 14.8 \%$ and $\triangle \mathrm{RDW}_{4-1} \geq 0.6 \%$ showed the longest survival time after standard treatment. At the same level of the Pneumonia Severity Index (PSI), patients with initial RDW $\leq 14.8 \%$ showed a tendency towards lower mortality than those with RDW $>14.8 \%$. In addition, patients with $\Delta \mathrm{RDW}_{4-1} \geq 0.6 \%$ tended to have lower mortality rates than those with $\Delta \mathrm{RDW}_{4-1}<0.6 \%$ at the same level of the PSI and the same initial RDW (initial RDW $\leq 14.8 \%$ : $6.8 \%$ vs $8.6 \%, p=0.74$; initial $\mathrm{RDW}>14.8 \%$ : $12.5 \%$ vs $36 \%$, $p<0.05)$. Following standardized admission, $\triangle \mathrm{RDW}$ showed a negative change, which can be attributed to the alleviation of inflammation and oxidative stress during the initial treatment of $\mathrm{CAP}^{36} \Delta \mathrm{RDW}$ can therefore be used as an independent predictor of short-term mortality in patients with CAP.

\section{$\triangle R D W$ and Chronic Obstructive Pulmonary Disease}

Chronic obstructive pulmonary disease (COPD) is a respiratory disease characterized by persistent airflow limitation, ${ }^{38}$ and the progression of this disease is associated with enhanced chronic inflammatory reflexes. Studies have shown a correlation between dynamic changes in RDW and COPD readmission rates. The 30day readmission rate was $27.1 \%$ in patients with a $\triangle \mathrm{RDW}$ positive change, ${ }^{39}$ and significantly higher than that in patients with $\triangle \mathrm{RDW}$ dynamic reduction (9.7\%). Monitoring the trend of $\triangle \mathrm{RDW}$ can play an essential role in the timely adjustment of COPD treatment options and assessment of prognosis.

\section{$\triangle R D W$ and Coronavirus Disease 2019}

Coronavirus disease 2019 (COVID-19) is a severe infectious disease, which is caused by severe acute respiratory syndrome coronavirus 2 (SARS-CoV-2). The global death toll for COVID-19 is about 490 million. Studies of patients with COVID-19 have shown that elevated RDW was correlated with decreased ventilator-free days in the intensive care unit (ICU). ${ }^{40}$ A progressive increase in RDW values was observed with advancing severity. ${ }^{41}$ RDW of $13.6 \%$ had a sensitivity of $80 \%$ and specificity of $59 \%$ for predicting mortality, while RDW of $14.5 \%$ (optimal cutpoint) had a sensitivity of $72 \%$ and specificity of $81 \%$ for predicting 30-day mortality. ${ }^{42}$ Survival analysis showed that patients with RDW $>13.0 \%$ had higher 30 -day mortality than patients with lower RDW. ${ }^{43}$

Patients with increased RDW during hospitalization had higher mortality compared to those with unchanged RDW: for normal RDW patients, mortality rose from $6 \%$ to $24 \%$, and for those with elevated RDW on admission, mortality rose from $22 \%$ to $40 \%{ }^{44}$ There is a continued relationship between RDW and mortality in all age groups, 
each $1 \%$ increment in RDW was associated with a $45 \%$ relative increase in mortality (HR 1.45, [1.30-1.60]), and that a $1 \%$ increase in RDW confers a similar mortality effect to a 10 -year increase in age. ${ }^{42}$

In a case report, the patient's RDW decreased continuously within 14 days of admission and increased gradually. ${ }^{45}$ Statistical analysis results showed that RDW was significantly lower than that of post-treatment. ${ }^{46}$ Whether the dynamic change of RDW is correlated with the progression of symptom severity still needs further study.

\section{$\Delta R D W$ and Sepsis with Disseminated Intravascular Coagulation}

Sepsis is usually defined as a systemic inflammatory syndrome caused by severe infection. As a marker reflecting the inflammatory process, ${ }^{47}$ RDW can show a significant increase in this disease. Multiple measurements of RDW in ICU patients revealed that RDW was positively correlated with patient mortality on any time point, and patients with a significant ascending trend of RDW in the first $72 \mathrm{~h}$ of admission were associated with a generally poor prognosis. $^{48} \Delta \mathrm{RDW}$ plays a positive role in clinical diagnosis and treatment. Coagulopathy may occur in 50\% to $70 \%$ of the patients with sepsis and is eventually complicated by disseminated intravascular coagulation (DIC) in approximately $35 \%$. The mean value of RDW in the DIC group was significantly higher than that in the non-DIC group $(15.73 \%$ vs $14.8 \%, p<0.01)$. Meanwhile, the mortality rate in the DIC group was much higher than that in the non-DIC group $(69.7 \%$ vs $30.1 \%, p<0.01)$, and the $\triangle \mathrm{RDW}$ was also significantly different ( 0.28 vs $0.11, p<$ $0.01){ }^{49} \Delta \mathrm{RDW}$ may be a strong predictor of DIC-related morbidity and mortality in septic patients.

\section{$\triangle$ RDW and Pulmonary Hypertension}

Pulmonary arterial hypertension (PAH) is a rare condition characterized by sustained elevation in pulmonary vascular resistance leading to right ventricular hypertrophy and end-stage right heart failure. ${ }^{50}$ Excluding the effect of age and gender, each standard unit (1.4\%) increase in RDW was related to the $90 \%$ higher odds of prevalent PAH. ${ }^{51}$ Although an association between $\triangle \mathrm{RDW}$ and $\mathrm{PAH}$ was suggested in this study, no causal relationship between $\triangle \mathrm{RDW}$ and disease development was observed in the investigators. It is unclear whether the $\triangle \mathrm{RDW}$ change occurred due to $\mathrm{PAH}$ hemolysis leading to polycythemia or other mechanisms that may have caused the RDW change. This requires further exploration in future studies.

\section{$\Delta R D W$ and Diabetes Mellitus}

During the past decade, the global prevalence of diabetes mellitus (DM) in the population has remarkably increased due to high intake of high-calorie food and diminished physical activity. RDW has been negatively associated with poor glycemic control. ${ }^{52}$ People with diabetes have distinctly higher RDW values than those without diabetes, each unit increase of RDW represents a $16 \%$ higher risk of incident diabetes. ${ }^{53}$ Higher RDW predicts a higher risk of diabetic complications, such as diabetic ketoacidosis (DKA) or diabetic retinopathy. ${ }^{54,55}$ This was due to the presence of micro- and macro-angiopathy with hyperglycemia that shortens the lifespan of RBCs. ${ }^{52}$ The association between $\triangle \mathrm{RDW}$ and diabetic complications should be further investigated.

\section{$\triangle R D W$ and Cardiovascular Disease}

Cardiovascular disease (CVD) is currently the leading cause of premature human death and disability globally, and its incidence is gradually increasing worldwide. In 2016, it was estimated that 17.9 million people died due to cardiovascular causes. ${ }^{56}$ The underlying pathogenesis and progression associated with almost all cardiovascular diseases are mainly due to the formation of atherosclerotic plaques in the coronary arteries, resulting in the development of coronary artery disease (CAD), cerebrovascular disease, venous thromboembolism, and peripheral vascular disease. Pilling et $\mathrm{al}^{57}$ obtained ideal laboratory test results for 469,104 participants, who were followed up for a period of 9 years. After excluding 228,627 anemic patients, the remaining 240,477 participants were prospectively analyzed. By the end of the study period, $\triangle \mathrm{RDW}$ strongly predicted the occurrence of diseases such as CAD (myocardial infarction or angina, $\mathrm{n}=5430$ ), heart failure $(\mathrm{n}=963)$, hypertension $(\mathrm{n}=10,615)$, atrial fibrillation $(n=3079)$, peripheral vascular disease $(n=728)$ and stroke (cerebrovascular disease, $n=1390$ ), indicating that $\triangle \mathrm{RDW}$ has a great predictive value in cardiovascular diseases. High RDW ( $\geq 15 \%$ variation, $n=6050$ ), compared to low $(<12.5 \% \mathrm{n}=20,844)$, was strongly associated with mortality (HR 3.10; 95\% CI: 2.57 to 3.74), and had $64 \%$ increased likelihood of a diagnosis $(\mathrm{HR}=1.64 ; 95 \%$ CI,1.49-1.81). Veeranna et $\mathrm{al}^{58}$ found that RDW 
(>12.6\%) was superior to $\mathrm{C}$-reactive protein (CRP) level in assessing CAD mortality within 6 years.

Lee et $\mathrm{al}^{59}$ investigated the relationship between changes in RDW after coronary artery bypass graft $(\mathrm{CABG})$ and the risk of postoperative morbidity. A total of 117 subjects were divided into an event-free group $(n=79)$ and event group ( $n=38)$ according to the occurrence of early adverse events. The results showed that women had a greater chance of adverse events, and those with adverse events had a lower body surface area; RDW increased significantly after CABG (pretreatment RDW: $13.10 \pm 0.95 \%$; post-treatment RDW: $13.42 \pm 0.95 \%$ ); when the $\triangle \mathrm{RDW}$ cut-off value was equal to 1.45 , the specificity for predicting early adverse events after CABG was $78.2 \%$, and the sensitivity was $71.1 \%(p<0.001)$. In addition, $\triangle \mathrm{CRP}$ and $\triangle \mathrm{WBC}$ were not significantly correlated with the occurrence of early adverse events.

In a study of 1242 patients with heart failure (HF), it was found that $\triangle \mathrm{RDW}$ was lower and stable in patients who survived within 30 days (RDW at admission: $15.3 \%$, RDW after 48 hours: 15.4\%, RDW after 96 hours 15.4\%), while this value was significantly increased in patients who died at 30 and 60 days (30-day mortality: RDW at admission: $16.3 \%$, RDW after 48 hours: $16.6 \%$, RDW after 96 hours: $17 \%, p<0.001$; 60 -day mortality: RDW at admission: $16.1 \%$, RDW after 48 hours: $16.3 \%$, RDW after 96 hours: $16.5 \%, \mathrm{p}<0.001)$. Therefore, it could be concluded that $\triangle \mathrm{RDW}$ was associated with both 30 -day and 60 -day mortality. It was also confirmed by KaplanMeier's survival analysis that both $\Delta \mathrm{RDW}>1 \%$ and $>0.4 \%$ after 96 hours of hospitalization were associated with an increased risk of death at 30 and 60 days of patients. ${ }^{60}$ Heart failure patients with increased $\triangle \mathrm{RDW}$ during hospitalization had significantly higher all-cause and cardiac-based mortality than patients with decreased levels. $^{61}$

In another study, 120 patients with acute myocardial infarction received routine treatment (including sedation, lipid-lowering, fluid infusion, oxygen inhalation, etc) for 1-2 weeks after thrombolytic therapy (including 61 patients with effective treatment and 59 patients with ineffective treatment). ${ }^{62}$ By analyzing RDW levels at admission and the end of the treatment cycle, it was found that the improvement of $\triangle \mathrm{RDW}$ in the treatmenteffect group was more significant than that in the ineffective group. The statistical analysis showed that the negative change of RDW could better reflect the therapeutic effect.
Other studies on cardiovascular diseases are listed below. Patients with decreased RDW or stable downward trend of $\triangle \mathrm{RDW}$ after cardiac resynchronization therapy (CRT) have a higher possibility of reversing left ventricular (LV) remodeling and survival. ${ }^{63}$ Geenen et $\mathrm{al}^{64}$ found that in patients who had completed atrial septal defect closure (ASD), RDW increased but changed less than high-sensitivity troponin-T (hs-TnT) and high-sensitivity C-reactive protein (hs-CRP) 1 day after ASD was closed. Within 3 months, $\triangle \mathrm{RDW}$ changed significantly but remained stable within 1 year and did not change significantly again. Possible explanations for this change were as follows: 1) myocardial injury due to device insertion and catheter device, 2) acute large left ventricular volume load due to myocardial injury induced by atrial septal defect closure after shunt cessation.

While many studies suggest a relationship between RDW and CVD, the pathophysiological mechanisms remain unclear. Whether high RDW levels directly affect cardiovascular diseases, or it is just a marker that surrogates something else remains to be answered?

Under certain pathophysiological conditions, changes in permeability reduce RBC deformability, leading to increased RDW levels, which causes low microvascular perfusion and ultimately makes cardiovascular disease more prominent. $^{65}$ Studies have shown that RBC deformability is reduced in microvascular disease when RDW levels exceed $14 \%{ }^{66}$ Chronic inflammation has also been the root cause of atherosclerosis and related complications. ${ }^{67}$ Pathological changes in red blood cell membranes with great free cholesterol can lead to accumulation of red blood cells in atheromatous plaques. ${ }^{68}$ Deposition of free cholesterol from the red blood cell membrane into atherosclerotic plaques will promote atherosclerosis, which propagates through the inflammatory cascade, ${ }^{69}$ thus providing a lipid-rich membrane for foam cells. On the other hand, a higher total cholesterol content in erythrocyte membrane is also one of the reasons for the deterioration of cell deformability, which can directly affect the lifespan of RBCs, and leads to accelerated cell turnover and increased RDW levels. ${ }^{70}$ In addition, alterations in lipids can even reduce the membrane fluidity of RBCs, leading to microcirculatory disturbance. In addition to the above possibilities, various mechanisms such as anemia, oxidative stress, and nutritional deficiency can also make a reasonable explanation. 


\section{Summary and Prospect}

By univariate analysis, higher RDWs predicted the occurrence of various common diseases and mortality in a group of healthy volunteers. When $\triangle \mathrm{RDW}$ increases within each interval, the risk of infection also increases. $\triangle \mathrm{RDW}$ can be chosen as a risk stratification tool to make patients with different RDW to be compared. Accurate risk stratification of the patients can effectively optimize resource allocation, thereby preventing overtreatment of lower-risk patients or inappropriate discharge of higher-risk ones, and ultimately improve clinical outcomes. In conclusion, $\triangle \mathrm{RDW}$ can be used as a marker of the severity in a variety of diseases and a predictor of comorbidities during hospitalization. And also, $\triangle$ RDW may be a useful marker to be included in health assessment (Table 1).

For $\triangle \mathrm{RDW}$, it is necessary to study further whether a stable change means a better prognosis. As a new index of risk stratification and prediction, $\triangle \mathrm{RDW}$ may be more instructive than a single index. A high RDW is also associated with many diseases, such as frailty syndrome, heart valve diseases, acute pancreatitis, Hodgkin lymphoma, and a variety of cancers. However, there are only few studies that have been conducted on the correlation between $\triangle R D W$ and clinical diseases by far. To testify whether it can be successfully applied in clinical practice, more large-scale studies are needed. Furthermore, a variety of biological indicators are high at the occurrence of a disease, play a role in the diagnosis of the disease, including white blood cells (WBC),

Table I Clinical Usefulness of $\triangle R D W$ in Human Disorders

\begin{tabular}{|l|l|}
\hline $\begin{array}{l}\text { The Unit Change of } \\
\text { RDW }\end{array}$ & $\begin{array}{l}\text { Changes in RDW Before and } \\
\text { After Treatment }\end{array}$ \\
\hline - Cardiac morbidities & - Weight indices after metabolic \\
Hypertension & surgery in patients \\
Coronary artery disease & - Breast cancer \\
Chronic heart failure & - Blood transfusion \\
Atrial fibrillation & - Rheumatoid arthritis \\
- Anemia & - Community-acquired pneumonia \\
- Chronic kidney disease & - Chronic obstructive pulmonary \\
- Chronic obstructive & disease \\
pulmonary disease & - Coronavirus disease 2019 \\
- Sleep apnea & \\
- Diabetes mellitus & \\
- Poor cognitive & \\
performance & \\
- Cerebral infarction & \\
- Blood transfusion & \\
- Coronavirus disease 2019 & \\
\hline
\end{tabular}

neutrophil-to-lymphocyte ratio (NLR), ${ }^{71}$ platelet-to-lymphocyte ratio (PLR), ${ }^{72} \mathrm{C}$-reactive protein (CRP), ${ }^{45}$ mean platelet volume (MPV), ${ }^{73}$ fibrinogen, ${ }^{74}$ and platelet distribution width (PDW), ${ }^{75}$ but the relationship between their changing trends has not received much attention.

This article introduces the dynamic changes and clinical significances in RDW of multiple diseases. The final purpose is to discuss whether it can be used for clinical diagnosis and research as an indicator of disease changes in a variety of systemic diseases. At present, the measurement methods of RDW are also different, and the definition criteria of $\triangle R D W$ have not been unified. If different centers can provide a unified standard for clinical practice, then the full value of $\triangle \mathrm{RDW}$ will be given. In addition, it would be helpful if the reference ranges of $\triangle \mathrm{RDW}$ in different diseases as well as in different ethnic groups could be determined. It is also important and interesting to explore whether $\triangle \mathrm{RDW}$ can be used as a long-term measure of disease change, similar to the role of glycosylated hemoglobin in assessing recent glycemic control.

\section{Funding}

This work was supported by Shandong Provincial Natural Science Foundation, China [Grant/Award Number: ZR2017MH097].

\section{Disclosure}

The authors report no conflicts of interest in this work.

\section{References}

1. Feng GH, Li HP, Li QL, Fu Y, Huang RB. Red blood cell distribution width and ischaemic stroke. Stroke Vasc Neurol. 2017;2(3):172-175. doi:10.1136/svn-2017-000071

2. Kenangil G, Ari B, Kaya F, Demir M, Domac F. Red cell distribution width levels in Parkinson's disease patients. Acta Neurol Belg. 2020;120(5):1147-1150. doi:10.1007/s13760-019-01197-1

3. Hammons L, Filopei J, Steiger D, Bondarsky E. A narrative review of red blood cell distribution width as a marker for pulmonary embolism. $J \quad$ Thromb Thrombolysis. 2019;48(4):638-647. doi:10. 1007/s11239-019-01906-w

4. Zhang F-X, Li Z-L, Zhang Z-D, Ma X-C. Prognostic value of red blood cell distribution width for severe acute pancreatitis. World J Gastroenterol. 2019;25(32):177-186. doi:10.3748/wjg.v25.i32.4739

5. Jia L, Cui S, Yang J, et al. Red blood cell distribution width predicts long-term mortality in critically ill patients with acute kidney injury: a retrospective database study. Sci Rep. 2020;10(1):1-11. doi:10. 1038/s41598-020-61516-y

6. Hu ZD, Lippi G, Montagnana M. Diagnostic and prognostic value of red blood cell distribution width in sepsis: a narrative review. Clin Biochem. 2020;77:1-6. doi:10.1016/j.clinbiochem.2020.01.001

7. Fan X, Deng H, Wang X, et al. Association of red blood cell distribution width with severity of hepatitis B virus-related liver diseases. Clinica Chimica Acta. 2018;482:155-160. doi:10.1016/j. cca.2018.04.002 
8. Hu GP, Zhou YM, Wu ZL, Li YQ, Ran PX. Red blood cell distribution width is an independent predictor of mortality for an acute exacerbation of COPD. Int J Tuberculosis Lung Dis. 2019;23 (7):817-823. doi:10.5588/ijtld.18.0429

9. Sotiropoulos K, Yerly P, Monney P, et al. Red cell distribution width and mortality in acute heart failure patients with preserved and reduced ejection fraction. ESC Heart Fail. 2016;3(3):198-204. doi:10.1002/ehf2.12091

10. Th AL, Jda R, Cunanan EL, Mda T, Fer P. Red cell distribution width and mortality in patients with acute coronary syndrome: a meta-analysis on prognosis. Cardiol Res. 2018;9(3):144. doi:10. $14740 / \mathrm{cr} 732 \mathrm{w}$

11. Subramanian M, Prabhu MA, Saravanan S, Thachathodiyl R. Biowidth index: a novel biomarker for prognostication of long term outcomes in patients with anaemia and heart failure. Acta Cardiol. 2017;73(4):403-409.

12. Xanthopoulos A, Papamichalis M, Zajichek A, et al. In-hospital red blood cell distribution width change in patients with heart failure. Eur J Heart Fail. 2019;21(12):1659-1661. doi:10.1002/ejhf.1546

13. Shao Q, Korantzopoulos P, Letsas KP, et al. Red blood cell distribution width as a predictor of atrial fibrillation. J Clin Lab Anal. 2018;32(5):e22378. doi:10.1002/jcla.22378

14. Giuseppe lippi GT, Montagnana M, Salvagno GL, Zoppini G, Guidi GC. Relation between red blood cell distribution width and inflammatory biomarkers in a large cohort of unselected outpatients. Arch Pathol Lab Med. 2009;133(4):628. doi:10.5858/133.4.628

15. He Y, Liu C, Zeng Z, Ye W, Lin J, Ou Q. Red blood cell distribution width: a potential laboratory parameter for monitoring inflammation in rheumatoid arthritis. Clin Rheumatol. 2018;37(1):161-167. doi:10.1007/s10067-017-3871-7

16. Kushang P, Ferrucci V, Ershler L, William B, Dan L. Red blood cell distribution width and the risk of death in middle-aged and older adults. Arch Intern Med. 2009;169(5):515. doi:10.1001/archinternmed.2009.11

17. Salvagno GL, Sanchis-Gomar F, Picanza A, Lippi G. Red blood cell distribution width: a simple parameter with multiple clinical applications. Crit Rev Clin Lab Sci. 2015;52(2):86. doi:10.3109/ 10408363.2014.992064

18. Li N, Zhou H, Tang Q. Red blood cell distribution width: a novel predictive indicator for cardiovascular and cerebrovascular diseases. Dis Markers. 2017;2017:1-23.

19. Xiao C-H, Wan J, Liu H, et al. Red blood cell distribution width is an independent risk factor in the prediction of acute respiratory distress syndrome after severe burns. Burns. 2019;45(5):1158-1163. doi:10. 1016/j.burns.2019.01.006

20. Shteinshnaider M, Barchel D, Almoznino-Sarafian D, Tzur I, Tsatsanashvili N. Prognostic significance of changes in red cell distribution width in an internal medicine ward. Eur J Intern Med. 2015;26(8):166-168.

21. Schmidt PJ, Ness PM. Hemotherapy: from bloodletting magic to transfusion medicine. Wiley Online Lib. 2006;46(2):166-168.

22. Spadaro S, Taccone FS, Fogagnolo A, et al. The effects of blood transfusion on red blood cell distribution width in critically ill patients: a pilot study. Transfusion. 2018;58(8):1863-1869. doi:10. $1111 /$ trf. 14759

23. Nielsen OJ, Andersen LS, Ludwigsen E, et al. Anaemia of rheumatoid arthritis: serum erythropoietin concentrations and red cell distribution width in relation to iron status. Ann Rheum Dis. 1990;49 (6):349-353. doi:10.1136/ard.49.6.349

24. Feng L, Wang X, Yan L, Liu D, Yang Z. Red blood cell distribution width in rheumatoid arthritis, ankylosing spondylitis and osteoarthritis: true inflammatory index or effect of anemia? Ann Clin Lab Sci. 2018;48(3):301-307.

25. Weuve J, Mendes D, Bennett DA, Dong X, Evans DA. The red cell distribution width and anemia in association with prevalent dementia. Alzheimer Dis Assoc Disord. 2017;28(2):99. doi:10.1097/WAD.0b $013 \mathrm{e} 318299673 \mathrm{c}$
26. Winchester LM, John P, Simon L, Nevado-Holgado AJ. Red blood cell indices and anaemia as causative factors for cognitive function deficits and for Alzheimer's disease. Genome Med. 2018;10(1):51. doi:10.1186/s13073-018-0556-Z

27. Beydoun MA, Hossain S, Maciver PH, Srinivasan D, Waldstein SR. Red cell distribution width, anemia, and brain volumetric outcomes among middle-aged adults. J Alzheimer's Dis. 2021;81:1-17.

28. Xia Y, Huimian C, He J, Che ZY. Association between red blood cell distribution width (RDW) and carotid artery atherosclerosis (CAS) in patients with primary ischemic stroke. Arch Gerontol Geriatrics. 2015;61(1):72-75. doi:10.1016/j.archger.2015.04.005

29. Hasan K, Selim D, Aysegul B, et al. Red cell distribution width and neurological scoring systems in acute stroke patients. Neuropsychiatr Dis Treat. 2015;11:733-739.

30. Kwang CY, Danai K, Anastasios D, et al. Inhibition of renin angiotensin axis may be associated with reduced risk of developing venous thromboembolism in patients with atherosclerotic disease. PLoS One. 2014;9(1):e87813. doi:10.1371/journal.pone.0087813

31. Kato H, Ishida J, Imagawa S, et al. Enhanced erythropoiesis mediated by activation of the renin-angiotensin system via angiotensin II type 1a receptor. FASEB J. 2005;19(14):2023-2025. doi:10.1096/fj.053820fje

32. Ashley C, Bradley R, Frazee W. Community-acquired pneumonia. Emerg Med Clin North Am. 2018;36(4):665-683. doi:10.1016/j. emc.2018.07.001

33. Nascimento arvalho CM. Community acquired pneumonia among children: the latest evidence for an updated management. Jornal de Pediatria (Versão Em Português). 2020;96:29-38. doi:10.1016/j. jpedp.2019.08.002

34. Leung AK, Wong AH, Hon KL. Community-acquired pneumonia in children. Recent Pat Inflamm Allergy Drug Discov. 2018;12 (2):136-144. doi:10.2174/1872213X12666180621163821

35. MandellLionel A. Community-acquired pneumonia: an overview. Postgrad Med. 2015;127(6):607-615. doi:10.1080/00325481.2015.107 4030

36. Ge Y, Liu C, Al-Masud M, Xiao R. Elevated Red blood cell distribution width combined white blood cell in peripheral blood routine have a better sensitivity than CURB-65 scores in predicting ICU admission and mortality in adult community-acquired pneumonia patients. Clin Lab. 2019;65(3). doi:10.7754/Clin. Lab.2018.180828

37. Lee SM, Lee JH, Kim K, You HJ, Park H. The clinical significance of changes in red blood cell distribution width in patients with community-acquired pneumonia. Clin Exp Emerg Med. 2016;3 (3):139-147. doi:10.15441/ceem.15.081

38. Celli BR, Wedzicha JA. Update on clinical aspects of chronic obstructive pulmonary disease. $N$ Engl J Med. 2019;381(13): 1257-1266. doi:10.1056/NEJMra1900500

39. Zhu M, Dai L, Wan L, Zhang S, Peng H. Dynamic increase of red cell distribution width predicts increased risk of 30-day readmission in patients with acute exacerbation of chronic obstructive pulmonary disease. Int J COPD. 2021;16:393-400. doi:10.2147/ COPD.S291833

40. Otero TM, Yeh DD, Bajwa EK, et al. Elevated red cell distribution width is associated with decreased ventilator-free days in critically ill patients. J Intensive Care Med. 2018;33(4):241-247. doi:10.1177/ 0885066616652612

41. Henry BM, Benoit JL, Benoit S, Pulvino C, Lippi G. Red Blood Cell Distribution Width (RDW) predicts COVID-19 severity: a prospective, observational study from the Cincinnati SARS-CoV-2 emergency department cohort. Diagnostics. 2020;10(9):618. doi:10. 3390/diagnostics10090618

42. Hornick A, Tashtish N, Osnard M, Shah B, Al-Kindi SG. Anisocytosis is associated with short-term mortality in COVID-19 and may reflect proinflammatory signature in uninfected ambulatory adults. Pathogens Immun. 2020;5(1):312-326. doi:10.20411/pai.v5i1.391 
43. Lorente L, Martín M, Argueso M, Solé-Violán J, Jiménez A. Association between red blood cell distribution width and mortality of COVID-19 patients. Anaesth Crit Care Pain Med. 2020;40 (1):100777. doi:10.1016/j.accpm.2020.10.013

44. Foy BH, Carlson JC, Reinertsen E, et al. Association of red blood cell distribution width with mortality risk in hospitalized adults with SARS-CoV-2 infection. JAMA Net Open. 2020;3(9):e2022058e2022058. doi:10.1001/jamanetworkopen.2020.22058

45. Lu G, Wang J. Dynamic changes in routine blood parameters of a severe COVID-19 case. Clinica Chimica Acta. 2020;508:98-102. doi:10.1016/j.cca.2020.04.034

46. Liang J, Nong S, Jiang L, et al. Correlations of disease severity and age with hematology parameter variations in patients with COVID-19 pre-and post-treatment. J Clin Lab Anal. 2021;35(1):e23609. doi:10.1002/jcla.23609

47. Horta-Baas G, Romero-Figueroa MD. Clinical utility of red blood cell distribution width in inflammatory and non-inflammatory joint diseases. Int J Rheum Dis. 2019;22(1):47-54. doi:10.1111/1756-185X.13332

48. Gupta M, Yadav G, Singh Y, Bhalekar A. Correlation of the changing trends of red cell distribution width and serum lactate as a prognostic factor in sepsis and septic shock. J Anaesthesiol Clin Pharmacol. 2020;36(4):531. doi:10.4103/joacp.JOACP_105_19

49. Fan YW, Liu D, Chen JM, Li WJ, Gao CJ. Fluctuation in red cell distribution width predicts disseminated intravascular coagulation morbidity and mortality in sepsis: a retrospective single-center study. Minerva Anestesiol. 2021;87(1):52-64. doi:10.23736/S03759393.20.14420-1

50. Stacher E, Graham BB, Hunt JM, et al. Modern age pathology of pulmonary arterial hypertension. Am J Respir Crit Care Med. 2012;186(3):261. doi:10.1164/rccm.201201-0164OC

51. Ulrich A, Wharton J, Thayer T, et al. Mendelian randomisation analysis of red cell distribution width in pulmonary arterial hypertension. Eur Respir J. 2019;55(2):1901486. doi:10.1183/13993003.01486-2019

52. Alamri B, Bahabri A, Aldereihim A, et al. Hyperglycemia effect on red blood cells indices. Eur Rev Med Pharmacol Sci. 2019;23 (5):2139-2150.

53. Wang J, Zhang Y, Wan Y, Fan Z, Xu R. The relationship between red blood cell distribution width and incident diabetes in Chinese adults: a cohort study. J Diabetes Res. 2020;27:1623247.

54. Atalay H, Boyuk B, Ates M, Guzel S, Celebi A, Ekizoglu I. Red cell distribution width and acute complications of diabetes. Acta Endocrinol. 2018;14(4):514. doi:10.4183/aeb.2018.514

55. Ma Y, Li S, Zhang A, et al. Association between red blood cell distribution width and diabetic retinopathy: a 5-year retrospective case-control study. J Ophthalmol. 2021;2021:6653969.

56. Evans MA, Sano S, Walsh K. Cardiovascular disease, aging, and clonal hematopoiesis. Ann Rev Pathol. 2020;15:419-438. doi:10.1146/annurev-pathmechdis-012419-032544

57. Pilling LC, Atkins JL, Kuchel GA, Luigi F, David M, Ru KY. Red cell distribution width and common disease onsets in 240,477 healthy volunteers followed for up to 9 years. PLoS One. 2018;13(9): e0203504. doi:10.1371/journal.pone.0203504

58. Veeranna V, Zalawadiya SK, Panaich S, Patel KV, Afonso L. Comparative analysis of red cell distribution width and high sensitivity C-reactive protein for coronary heart disease mortality prediction in multi-ethnic population: findings from the 1999-2004 NHANES. Int $J$ Cardiol. 2013;168(6):5156-5161. doi:10.1016/j. ijcard.2013.07.109

59. Lee SI, Lee SY, Choi CH, Park C-H, Park KY, Son KH. Relation between changes in red blood cell distribution width after coronary artery bypass grafting and early postoperative morbidity. $J$ Thorac Dis. 2018;10(7):4244. doi:10.21037/jtd.2018.06.108
60. Turcato G, Zorzi E, Prati D, et al. Early in-hospital variation of red blood cell distribution width predicts mortality in patients with acute heart failure. Int J Cardiol. 2017;243:306-310.

61. Uemura Y, Shibata R, Takemoto K, et al. Elevation of red blood cell distribution width during hospitalization predicts mortality in patients with acute decompensated heart failure. $J$ Cardiol. 2016;67 (3):268-273. doi:10.1016/j.jjcc.2015.05.011

62. Jian Y, Li W, Peng Y, Xiong M, Zhang M. Dynamic Monitoring of Erythrocyte Distribution Width (RDW) and Platelet Distribution Width (PDW) in treatment of acute myocardial infarction. Medical Sci Monitor Int Med J Exp Clin Res. 2017;23:5899-5906.

63. Carluccio E, Biagioli P, Alunni G, et al. Non-cardiac factors for prediction of response to cardiac resynchronization therapy: the value of baseline, and of serial changes, in red cell distribution width. Int $J$ Cardiol. 2017;243:347-353. doi:10.1016/j.ijcard.20 17.05.123

64. Geenen LW, de Assis LU, Baggen VJ, et al. Evolution of blood biomarker levels following percutaneous atrial septal defect closure in adults. IJC Heart Vasculature. 2020;30:100582. doi:10.1016/j. ijcha.2020.100582

65. Reinhart WH, Piety NZ, Goede JS, Shevkoplyas SS. Effect of osmolality on erythrocyte rheology and perfusion of an artificial microvascular network. Microvasc Res. 2015;98:102-107. doi:10.1016/j. mvr.2015.01.010

66. Patel KV, Mohanty JG, Kanapuru B, Hesdorffer C, Ershler WB, Rifkind JM. Association of the red cell distribution width with red blood cell deformability. Adv Exp Med Biol. 2013,765:211-216. doi:10.1007/978-1-4614-4989-8_29.

67. Zyga S, Kolovos P. Cardiovascular disease and chronic inflammation in end stage kidney disease. Int J Caring Sci. 2013;6(1):29-36.

68. Tziakas DN, Chalikias GK, Boudoulas H. Significance of the Cholesterol Content of Erythrocyte Membranes in Atherosclerosis. Taylor \& Francis; 2010:449-452.

69. Chalikias G, Kikas P, Drosos I, et al. Sphingomyelin content of erythrocyte membranes is in linear association with membranes' loading with cholesterol in coronary artery disease patients. Atherosclerosis. 2016;252:e119-e120. doi:10.1016/j.atherosclerosis. 2016.07.634

70. Vayá A, Sarnago A, Fuster O, Alis R, Romagnoli M. Influence of inflammatory and lipidic parameters on red blood cell distribution width in a healthy population. Clin Hemorheol Microcirc. 2015;59 (4):379-385. doi:10.3233/CH-141862

71. Han F, Liu Y, Cheng S, Sun Z, Ju Y. Diagnosis and survival values of neutrophil-lymphocyte ratio (NLR) and red blood cell distribution width (RDW) in esophageal cancer. Clinica Chimica Acta. 2018;488:150-158.

72. Wu J, Zhang X, Liu H, Guo N, Pan Q, Wang Y. RDW, NLR and RLR in predicting liver failure and prognosis in patients with hepatitis E virus infection. Clin Biochem. 2019;63:24-31. doi:10.1016/j. clinbiochem.2018.11.012

73. May JE, Marques MB, Reddy VVB, Gangaraju R. Three neglected numbers in the CBC: the RDW, MPV, and NRBC count. Cleve Clin $J$ Med. 2019;86(3):167-172. doi:10.3949/ccjm.86a.18072

74. Wang X, Wu CY, Zeng PY, Li LL, Chai Y. Value of red blood cell distribution width and fibrinogen level for evaluation of the therapeutic efficacy and prognosis in patients with diffuse large B-cell lymphoma. Zhongguo Shi yan Xue ye Xue Za Zhi/Zhongguo Bing li Sheng Li Xue Hui/J Exp Hematol/Chin Assoc Pathophysiol. 2020;28 (1):153-159.

75. Ahin F, Koar AF, Aslan AF, Yiitba B, Uslu B. Serum biomarkers in patients with stable and acute exacerbation of chronic obstructive pulmonary disease: a comparative study. J Med Biochem. 2019;38 (4):503-511. doi:10.2478/jomb-2018-0050 


\section{Publish your work in this journal}

The International Journal of General Medicine is an international, peer-reviewed open-access journal that focuses on general and internal medicine, pathogenesis, epidemiology, diagnosis, monitoring and treatment protocols. The journal is characterized by the rapid reporting of reviews, original research and clinical studies across all disease areas. The manuscript management system is completely online and includes a very quick and fair peer-review system, which is all easy to use. Visit http://www.dovepress.com/ testimonials.php to read real quotes from published authors.

Submit your manuscript here: https://www.dovepress.com/international-journal-of-general-medicine-journal 